\title{
Les pratiques BDSM queer et le fist-fucking dans Quatrième génération et Insurrections ! en territoire sexuel de Wendy Delorme
}

\section{Christina CHUNG, Université de Toronto}

Écrivaine, performeuse et activiste LGBTQ+ française, Wendy Delorme a publié plusieurs ouvrages depuis 2007, notamment le roman Quatrième génération (2007), le recueil d'essais Insurrections! en territoire sexuel (2009), le recueil de nouvelles In/soumises : contes cruels au féminin (2012) et le roman La Mère, la Sainte et la Putain (2012). Dans le cadre de mon analyse, je me penche uniquement sur les deux premiers textes ${ }^{1}$, parce qu'ils traitent des pratiques BDSM et de la valeur politique de ces pratiques. Comme Marion, narratrice de Quatrième génération, l'affirme, «la sodomie c'est la vie, la transphobie tue, pour claquer le pavé en bottes de combat ou en talons aiguilles, pour vivre avec rage et force et joie, et pour s'envoyer en l'air parce que chacun de nos orgasmes c'est un défi lancé à la face du monde » (321; l'auteure souligne). D'ailleurs, le titre du roman est l'affirmation de l'existence de la quatrième génération de féministes, qui ne se veut pas être en rupture avec la troisième, mais plutôt en continuation avec un mouvement permettant les libertés individuelles tout en prenant position par son corps, tel que soutenu par le mouvement post-pornographie (Ausina 94). D’une part, l'hétéronormativé se définit par un ensemble de normes ayant pour effets de naturaliser la notion du couple hétérosexuel, de promouvoir la reproduction sexuelle et de dévaluer ce qui s'en éloigne. D'autre part, elle pathologise, criminalise et condamne les façons d'être queer, telles que l'homosexualité, la bisexualité, le sexe BDSM, la promiscuité et le sexe commercial, entre autres (Bauer 2). Quant aux textes de Delorme, ils célèbrent la non-hétéronormativité, la diversité des sexualités et des identités de genre. Dans Quatrième génération, par exemple, la narratrice enrichit le dialogue féministe, à la fois en exprimant sa réflexion et ses positionnements intellectuels et en agissant directement, c'est-à-dire en narrant en détail ses relations avec des personnes queer. Ce qui est intéressant chez Delorme, c'est surtout la représentation des pratiques BDSM queer ${ }^{2}$. Cette auteure démystifie les stéréotypes associés aux personnes queer et aux pratiques non hétéronormatives. Pour mettre en relief la manière dont elle représente les pratiques BDSM queer, j'ai divisé mon analyse en cinq parties : premièrement, j'illustre comment l'ondinisme, soit la pratique sexuelle qui repose sur une érotisation de l'urine, s'inscrit dans les pratiques BDSM queer ; j'examine, lors 
des deux autres parties, le lexique et les stratégies textuelles utilisés pour représenter le BDSM et les techniques nécessaires ; enfin, dans les quatrième et cinquième parties, j'analyse deux extraits mettant en avant le fist-fucking. Mais, auparavant, faisons un survol des études critiques liées aux pratiques BDSM.

\section{Les luttes pour les pratiques BDSM}

Dans son chapitre «Thinking Sex », Gayle Rubin rappelle que, même avant la publication de l'essai «Sadisme et masochisme » (1905) de Freud - qui pathologise le sadomasochisme -, à la fin du dix-neuvième siècle, il y avait des campagnes éducatives et politiques en Angleterre et aux États-Unis qui encourageaient la chasteté et l'élimination de la prostitution et qui décourageaient la masturbation (138). Rubin ajoute que la religion chrétienne contribue à l'oppression sexuelle car celle-ci considère que les pratiques sexuelles sont impures en dehors de leur fonction procréatrice (148). L'anthropologue démontre en outre que, dans les sociétés occidentales modernes, les pratiques sexuelles sont hiérarchisées. Elle illustre ce fait à l'aide d'une pyramide érotique. Selon elle, au sommet de cette pyramide se situent les hétérosexuel·le·s qui sont marié·e.s et assument leur responsabilité reproductive (149). S'ensuivent les minorités sexuelles telles que les transsexuel·le·s, les travesti·e.s, les fétichistes, les sadomasochistes et les travailleur-euse-s du sexe. Finalement, au bas de la pyramide se retrouvent celles et ceux dont l'érotisme transgressent les frontières générationnelles (149). Rubin met également l'accent sur la différence entre le mouvement antipornographie et le féminisme pro-sexe. Elle démontre que le mouvement antipornographie, mené par certaines féministes telles que Catharine MacKinnon et Andrea Dworkin, a tendance à contribuer à l'oppression de la sexualité des femmes au lieu de leur libération sexuelle étant donné que ces féministes accusent d'antiféminisme les femmes dont les pratiques sexuelles se situent au bas de la pyramide (Rubin 172). Quant au féminisme pro-sexe, il revendique une sexualité non conformiste, et est mené particulièrement par les lesbiennes sadomasochistes, les gouines butches/fems, les hétérosexuel·le·s qui ne souscrivent pas à l'idéologie de reproduction et les féministes radicales (173-174). Somme toute, selon Rubin, tant le mouvement pro-sexe qu'une théorie radicale du sexe sont nécessaires pour identifier, décrire, expliquer et dénoncer l'injustice érotique et l'oppression sexuelle.

Pat Califia, auteur de Public Sex : The Culture of Radical Sex (1994), présente la notion du «vanilla sex » afin de légitimer la pratique sadomasochiste. D’une certaine façon, il conteste 
l'hégémonie du «vanilla sex », le seul qui soit considéré comme acceptable dans la société occidentale. Le « sexe vanille » est, selon Califia, la pratique sexuelle qui encourage la douceur. Il soutient que cette pratique est normative puisqu'elle positionne les femmes en tant que dociles et passives, à l'opposé des femmes sexuellement actives qui constituent supposément une menace pour la société (157). En effet, il est possible pour celles-ci de revendiquer leur « activité » sexuelle en participant au sadomasochisme, une pratique située aux antipodes du sexe vanille et considérée comme agressive par les féministes antipornographie. Califia insiste sur le fait que le sadomasochisme n'est pas une forme d'agression sexuelle, mais plutôt une activité consensuelle qui inclut des rôles polarisés et des sensations intenses (168). Il soutient que si le sadomasochisme est persécuté, c'est en partie parce que les rôles adoptés dans le cadre de cette pratique défient les catégories de genre, d'orientation sexuelle, de race et de classe sociale (163). Il en découle une inintelligibilité qui rend cette pratique inconcevable aux yeux de la société dominante. Selon Califia, les médias, tels que la diffusion de la pornographie commerciale, déforment la représentation des pratiques BDSM : « [commercial pornography] is designed to make money, not to educate people or to be aesthetically pleasing » (172). Le peu de pornographie S/M, qui est informatif et est bien produit, risque d'être éliminé à cause du mouvement antipornographie (173). Califia considère que l'abolition de la pornographie $\mathrm{S} / \mathrm{M}$ pourrait à la fois appauvrir la culture $\mathrm{S} / \mathrm{M}$ et isoler les sadomasochistes (173). En outre, les membres du mouvement antipornographie ont accusé le sadomasochisme de faire violence aux femmes en les cantonnant dans des positions de soumission (173). Califia défend les pratiques sadomasochistes et affirme, comme Rubin, que c'est en interdisant les pratiques sexuelles minoritaires que l'oppression des femmes est accentuée. De nos jours, même au vingt et unième siècle, le sadomasochisme est encore un sujet tabou, en grande partie en raison des discours médicaux, religieux et antipornographie.

Afin d'éclairer les mythes qui entourent le sadomasochisme, Jessica Caruso fait une analyse approfondie des pratiques BDSM dans son livre BDSM : les règles du jeu (2016). D'abord, elle souligne que les membres de la communauté sadomasochiste préfèrent utiliser le terme «BDSM » plutôt que « SM », car il englobe une plus grande diversité de pratiques, telles que le bondage et la discipline, la domination et la soumission, en plus du sadomasochisme (25). Ensuite, Caruso décrit ce que le BDSM n'est pas :

le BDSM exclut la violence, la coercition, la cruauté et le non-consentement ${ }^{3}$. Le BDSM n'est pas non plus de l'abus ou de l'exploitation et n'implique jamais d'enfants ou d'animaux. Même si des mots qui ont généralement une connotation 
négative sont utilisés (par exemple, l'humiliation, le contrôle, la punition), la personne qui reçoit a toujours préalablement consenti au type de pratique qu'elle va « subir », sachant qu'il revient au Dominant d'en choisir le moment et la méthode. Par ailleurs, les motivations qui sous-tendent les différentes pratiques ne devraient jamais être négatives (par exemple, la vengeance, la colère ou la haine). (29) ${ }^{4}$

Cette description s'oppose à l'idée que s'en faisait le mouvement antipornographie. Les adeptes du BDSM font aussi ressortir des motivations de plusieurs ordres, « dont les plus saillantes sont le plaisir, le sentiment d'être connecté à son partenaire, le fait d'échapper au quotidien, et parfois l'expression irrépressible de leur identité » (39). Dans Quatrième génération et Insurrections ! en territoire sexuel, les pratiques BDSM sont justement représentées de manière à ce que ces motivations soient mises en valeur.

\section{L'ondinisme ${ }^{5}$ comme expérience intime}

Dans son étude sur le BDSM queer, Robin Bauer affirme que les pratiques BDSM créent des situations ou des expériences intimes. Il ne définit pas l'intimité dans le sens d'amour, mais comme une situation intense, de partage et de transgression de limites (110), surtout lorsque des «personal boundaries are touched or crossed in some way » (111). Selon lui, la familiarité entre des partenaires - qui se connaissent depuis longtemps - donne lieu à une forme d'intimité, mais en performant des jeux de rôle, des éléments d' « unfamiliarity » sont réintroduits dans la relation (124). Durant un jeu de rôle qui implique l'objectification d'une personne, par exemple, celle-ci, en incarnant un objet, perd sa familiarité et devient étrange ( « unfamiliar ») vis-à-vis du partenaire étant donné que ses traits humains sont déniés. Paradoxalement, Bauer écrit que «intimacy is reenabled in the sense of boundary transgression and touch » durant le jeu car, quand les partenaires se touchent, ils surmontent le sentiment d'étrangeté qu'a créé la personne objectifiée (124). Ainsi, une situation intime est reproduite dans la relation. Delorme met cette forme d'intimité en scène dans son premier roman lorsque deux personnages - l'un jouant le rôle de la top ${ }^{6}$ et l'autre, celui du bottom - vivent une nouvelle expérience qui leur permet de transgresser leurs limites. Toilet, l'un des personnages, est le client de Corie, une travailleuse du sexe. Celle-ci est accompagnée de Marion, qui a consenti à aller chez Toilet pour son premier travail du sexe. Avant sa rencontre avec Toilet, Marion exprime un malaise : «Corie m'a téléphoné et c'est là que j'ai réalisé que j'avais complètement oublié de boire trois litres d'eau après le dîner » (MVT, 68-69). Cette angoisse est soulignée par la répétition du verbe « oublier » et par la longue phrase qui suit : 
J'avais oublié de boire trois litres d'eau juste après les raviolis thaïlandais parce que j'avais oublié que ce soir Corie et moi on va chez Toilet, un client de Corie, ce qui veut dire que je vais devoir pisser trois litres d'eau dans la bouche de Toilet, ce qui est ennuyeux puisque j'ai oublié de boire trois litres d'eau. (MVT, 69 ; je souligne)

On remarque le fait que le sentiment d'inconfort, illustré par l'adjectif «ennuyeux », n'est pas causé par le fait de devoir «pisser trois litres d'eau», mais bien d'avoir oublié de boire suffisamment pour livrer ce qui est attendu d'elle. En outre, en utilisant le registre populaire, Marion banalise la situation, comme s'il était habituel d'uriner dans la bouche de quelqu'un, qui plus est, un étranger - bien que ce soit la première fois qu'elle fasse ce travail. Il y a donc une rupture entre l'attente du lectorat - qui pourrait être surpris par ce genre de pratique - et la situation vécue par la narratrice. Le travail ne gêne pas Marion ; c'est la préparation qui est exigeante. Les verbes au plus-que-parfait montrent aussi que la condition à remplir ne peut se faire instantanément. La difficulté à se préparer avant une scène BDSM est observée dans un autre passage, où Delorme use de la répétition «trois litres d'eau » et énumère des actions, accompagnées de l'adjectif « impossible » : «j'avais très exactement 20 minutes pour boire trois litres d'eau, faire des retouches à mon maquillage et me changer, ce qui m'était matériellement impossible »(70). Cette même difficulté est amplifiée quand la narratrice emploie l'adverbe «très » et la répétition de «trois litres d'eau »: «C'est très difficile de calculer le bon timing quand il s'agit de pisser trois litres d'eau sur quelqu'un » (70). Comme le définit Bauer, une scène BDSM est une expérience intime, mais la rencontre entre Marion et Toilet s'avère plutôt similaire à ce que Bauer nomme un théâtre intime (intimate theater) étant donné que la performeuse - dans ce cas-ci, Marion - imagine la scène au préalable et appréhende d'être incapable de faire son travail comme il se doit ${ }^{7}$.

Au moment où Marion se trouve chez Toilet pour effectuer son travail, on observe, comme le prétend Bauer, un sentiment d'étrangeté qui établit une situation intime. Outre les difficultés rencontrées lors de la préparation, Marion souligne qu'elle doit surmonter certains obstacles durant la scène BDSM. D'abord, elle pense être incapable d'uriner en raison de la nature du travail ; uriner dans ce cas-ci devient une tâche, alors qu'habituellement, l'action est un besoin naturel. Ensuite, elle doit veiller à ce que son incapacité ne se traduise pas en incompétence, surtout devant Corie et Toilet : «Je me sens rougir. Ça m’énerve qu'elle [Corie] puisse penser que je suis intimidée. Ça m'énerve encore plus de penser que le type puisse penser ça. Je n'ai aucun problème à pisser en public ou à pisser debout. Je l'ai fait des centaines de fois. Mais là on me paye pour ça et j'y arrive 
pas » (76). Après quelques minutes, Marion parvient à se vider « goutte à goutte puis en rafales » (76). La narratrice conclut que ce n'est pas les technicités liées aux préparatifs qui retenaient son urine, mais bien l'excitation sexuelle : «Moi je sais pourquoi je n'ai pas réussi du premier coup : l'excitation sexuelle empêche de pisser, et je suis bel et bien excitée par la scène » (76). Une fois de plus, il y a une rupture entre ce qui est attendu de la lecture du texte et ce que la narratrice réfute par la suite. Les aventures qu'elle vit deviennent des occasions d'auto-découverte puisqu'elle acquiert, ce faisant, une meilleure compréhension de son propre corps. Avec Toilet, par exemple, Marion se découvre une capacité de créer une expérience intime avec lui tout en transgressant sa limite d'uriner lors d'une scène BDSM. Cette intimité est d'ailleurs partagée par Toilet qui, lui, ressent du plaisir à engloutir l'urine de Marion : «il ouvre grand la bouche, creusant les joues et poussant les lèvres en avant à chaque goulée » (76). Dans cette scène BDSM, le plaisir de Toilet est manifeste, et il convient à présent d'analyser la manière dont cette sensation est liée à sa désubjectivation.

Marion ne révèle à aucun moment le vrai prénom de son client, auquel elle réfère également en le désignant par « le type »: «ça m’énerve encore plus de penser que le type puisse penser ça », «je décide d'aller m'asseoir sur le trépied au-dessus du visage du type » (76). En employant «type »- mot définissant un homme quelconque - la narratrice opère une mise à distance entre elle et Toilet. Ce surnom a pour effet de signaler un manque d'intimité sans pour autant dénier le caractère humain du personnage masculin. Ce dernier est effectivement privé de sa singularité, sa subjectivité est déniée, mais il n'est pas pour autant réduit à proprement dit au statut de chose ou d'objet. En ce sens, il n'y a ni chosification ni objectification en soi. En revanche, on note une désubjectivation. Ce procédé constitue une partie intégrante du scénario BDSM où, dans cette scène précise, la narratrice joue le rôle d'une personne qui urine et le client, celui d'une toilette. À la lumière de la théorie de Bauer, le client vit une situation intime durant laquelle il expérimente la vulnérabilité par la désubjectivation. Similairement aux propos de Bauer, Caruso affirme que «[1]es jeux d'urologie et de scatologie qui consistent à uriner ou à déféquer sur le corps ou le visage du soumis comportent presque toujours un élément de dégradation » (95). Ce jeu procure du plaisir à Toilet et n'est pas représenté de manière négative dans le roman. En effet, après s'être fait uriner dessus, son sourire «gentil » $(\mathrm{MVT}, 74)$ et «doux » (78) manifeste un sentiment de sérénité. D’autres passages illustrent cette pratique BDSM, qui repose sur la soumission de Toilet pendant le jeu : «La dernière gorgée avalée, Toilet prend sa respiration et murmure : "Merci, 
Maîtresse" [à Corie] » (75), « Toilet murmure un autre "Merci Maîtresse” [à Marion] » (77). Ce n'est pas uniquement l'action de murmurer qui indique la soumission de Toilet face à Corie et à Marion, mais c'est aussi le lexique employé envers les deux dominatrices. En mettant en scène cette pratique sexuelle, Delorme démystifie le BDSM en contestant le préjugé selon lequel il s'agit de pratiques violentes et néfastes pour les adeptes. Au contraire, ces derniers en ressortent avec un sentiment de bien-être et vivent des expériences intimes et singulières. Comme nous l'avons vu chez Rubin, la soumission durant les scènes BDSM ne contribue pas à l'oppression, mais bien à une libération sexuelle. C'est justement cette libération dont nous sommes témoins chez Delorme.

\section{La représentation lexicale des pratiques BDSM}

Hormis le chapitre portant sur l'ondinisme, on retrouve dans Quatrième génération les pratiques BDSM les plus connues, telles que les jeux de rôle de domination/soumission. Marion narre une scène où l'une de ses partenaires, Dayne ${ }^{8}$, la «manipule comme une poupée » (PFL, 122). À la suite de cette séance, Marion exprime un sentiment de bien-être, mis en valeur à travers l'emploi de l'antithèse : «J'ai failli pleurer comme souvent après la jouissance mais ça avait été une telle libération cet orgasme que j'étais juste bien » (122; je souligne). Cette figure de style a pour effet d'illustrer l'existence de caractéristiques humaines, en l'occurrence les sentiments de Marion après qu'elle a été temporairement objectifiée en poupée. Cette scène souligne aussi que les pleurs ne sont pas causés par la douleur, mais bien par le bonheur. Il serait judicieux de dire que Delorme démystifie les sentiments ressentis par les soumis, comme par Marion, lors d'une séance de BDSM car, conformément aux propos de Califia évoqués ci-haut, les pratiques BDSM ne sont pas une forme d'agression, mais une activité qui inclut des sensations intenses. Un peu plus loin dans le roman, la narratrice se retrouve avec une autre partenaire, Karl, et elles sont toutes les deux entourées d'autres amies qui s'adonnent à une séance BDSM : «[Karl] a trouvé l'angle parfait, et m'a baisée à coups de poing pendant un long moment, en regardant Toni se branler sous les coups de canne qu'Irène appliquait sur ses cuisses et ses fesses. Je n'ai pas réussi à jouir, j’y arrive rarement quand on me baise à quatre pattes, mais je me sentais de bien meilleure humeur » (162 ; je souligne). Il est intéressant d'observer que le texte détourne la sexualité de sa finalité orgasmique. En effet, ce qui est mis de l'avant est l'accumulation des actions des personnages féminins qui se donnent du plaisir. À travers cet extrait, Delorme suggère que l'orgasme n'est pas nécessaire pour ressentir du plaisir car, comme c'est le cas pour Marion, toute l'activité sexuelle 
peut améliorer l'humeur. Dans le chapitre «Single Tail », le même sentiment de bien-être, à la suite d'une séance BDSM, est présent. Si le single tail, un fouet en cuir, est dans certains contextes un outil de punition pour des humains ou un outil de dressage pour les animaux, dans le cadre des pratiques BDSM, il constitue un instrument parmi d'autres. Certes, il est utilisé pour infliger de la douleur, mais celle-ci est souhaitée par la personne fouettée. Marion souligne les bienfaits de sa séance BDSM avec une amie, Tara, qui la fouette à l'aide d'un single tail :

Mais d'abord elle me fait asseoir à ses pieds pour que je délace ses bottes, parce qu'elle aime être pieds nus pour fouetter. Je suis en apnée. Le calme de ses gestes est lénifiant. [...] Je suis en train de sombrer dans cet espace de mon cerveau qui est déconnecté de la réalité, je ne peux plus parler. Chaque mot prononcé me ramènerait à la surface et je veux rester plongée dans cette partie de moi sombre et reposante, n'entendre que les claquements des fouets et la voix de Tara, ne pas penser (ST, 86-87; je souligne).

Ainsi, ce n'est pas tant la douleur' ${ }^{9}$ elle-même qui procure un sentiment de bien-être, mais la déconnexion d'avec la réalité et d'avec le Symbolique, dans le sens où les mots ne sont pas bienvenus. Un autre système de communication se met en place. À cet égard, Bauer compare les vertus des pratiques BDSM à des pratiques spirituelles :

Receiving intense sensations in a dyke+queer BDSM setting also enabled some interview partners to refocus through letting go of their mundane worries. The intense stimulation of the body may clear the head and enable one to stay completely focused in the moment. Many spiritual practices, such as meditation, are also aimed at reaching this state of being. It may, therefore, not be surprising that, for many interview partners, BDSM sessions turned into spiritual experiences, and some BDSM sessions were planned with the intent to enter a spiritual space (167).

Chez Delorme, cette importance accordée à la valeur spirituelle, voire thérapeutique, des pratiques BDSM, est révélée grâce au lexique. Après avoir reçu vingt coups de fouet, Marion exprime son état psychologique : «Je suis parfaitement calme, parfaitement concentrée, parfaitement shootée à l'adrénaline » (ST, 87 ; je souligne). Notons la répétition du mot «parfaitement » qui accompagne l'énumération des adjectifs et qui suggère les effets positifs, voire l'atteinte d'un nirvana, à partir de la séance de fouet. Si l'auteure de Quatrième génération met en avant l'intensité des sensations, j'analyse dans la prochaine partie la manière dont elle tente de montrer que la pratique BDSM exige de l'habileté. 


\section{Le BDSM comme compétence}

Lors de certaines séances BDSM, les adeptes doivent avoir une excellente connaissance du maniement des instruments, comme le fouet, afin d'assurer leur bon déroulement. Le gang bang, terme populaire désignant généralement des rapports sexuels menés par plusieurs partenaires consentants et centrés simultanément sur une seule personne, est un exemple de séance BDSM qui requiert à la fois des objets spécifiques et une grande habileté. Un chapitre d'Insurrections! en territoire sexuel exemplifie une telle pratique en mettant en scène des femmes anonymes, dont l'une est la narratrice autodiégétique. Avant de passer à l'action, celle-ci s'assure d'avoir tous les accessoires dont elle aura besoin pour la séance : «Les gants de latex noir et le lubrifiant sans silicone ni glycérine, les capotes et deux butt plug, un assortiment de pinces à seins, des dentelées et des plates, et bien entendu des digues dentaires noires et des roses très fines et douces au toucher, vive le latex » $(\mathrm{GB}, 65)$. En énumérant les instruments et les accessoires de protection, la narratrice insiste sur des détails essentiels à la préparation d'une séance BDSM. À travers les mots de l'instance narrative, Delorme se fait pédagogue en soulignant l'importance de la protection contre les infections transmissibles sexuellement et par le sang (ITSS). Durant une séance de gang bang, chaque individu est exposé à de nombreux risques et il leur faut, avant toute chose, obéir à la directive de la communauté BDSM, connue sous l'acronyme SSC - Safe, Sane and Consensual (Bauer 145). La question du «safe » concerne non seulement les accessoires utilisés pendant une séance BDSM, mais aussi la précision des actes qu'implique la pratique sexuelle. Dans la citation suivante, les virgules et les points sont justement utilisés pour illustrer chaque étape de la séance entre la narratrice et ses partenaires :

On me fesse à quatre mains, on fait glisser la fermeture de ma robe pour l'ouvrir, on remonte mon jupon, on décroche les bas de mon porte-jarretelles, on descend la culotte en dentelle jusqu'à mes chevilles mal assurées sur leurs talons aiguilles. On passe une main glissante et froide, enduite de lubrifiant, entre mes fesses, on me tire les cheveux, on m'enfonce un long gode lisse dans ma chatte, on me met un doigt dans le cul, on m'arrache le foulard d'entre les dents pour forcer mes lèvres consentantes avec un autre gode, qui distend mes mâchoires. Ma nuque se raidit sous l'effort, mes cuisses tremblent, on fait glisser un vibromasseur le long de mon échine, on l'applique sur mon sexe par-dessous le cheval d'arçons, on me traite de pute et de salope, on me dit que j'aime ça, on me fait jouir (GB, 66-67).

Il est possible de distinguer trois étapes, chacune séparée par un point. La première partie figure une sorte de foreplay, soit la phase de préparation amenant la narratrice à l'excitation sexuelle. Suit la deuxième partie durant laquelle la pénétration a lieu. La troisième partie s'attache au 
moment où la narratrice jouit. À travers l'utilisation des virgules, on a l'impression de suivre le rythme de chaque action, qui s'enchaîne rapidement. Comme Caruso l'évoque dans son étude sur les pratiques BDSM, la soumise a préalablement consenti à la pratique sexuelle ; elle n'est pas agressée, mais ressent du plaisir (29). Les dominantes, quant à elles, semblent avoir déjà calculé leur méthode d'avance et respectent un ordre spécifique de leurs actions. Cette méthode est représentée textuellement par le biais de la figure de l'amplification, qui emploie plus spécifiquement l'anaphore du sujet «on» et la gradation ascendante des verbes «tire », « enfonce », « arrache » et «forcer ». Enfin, ce sont toutes des actions précises qui mènent à la jouissance de la narratrice.

La narratrice de Quatrième génération souligne aussi la nécessité que les adeptes du BDSM soient précis, particulièrement dans un chapitre où elle participe à une fête dont le thème est la Rome antique. Alors que son amie, Corie, joue le rôle du maître, Marion est l'esclave ${ }^{10}$ punie. Cette scène décrit Corie de manière à représenter son habileté : «Corie est très douée pour les fessées à la canne et c'est une canne dont elle se sert ce soir, pour nous punir Spartacus et moi d'être de si mauvais esclaves [...]. Elle est très habile aussi en termes d'humiliation verbale » (CP, 107 ; je souligne). La répétition de l'adverbe «très », suivie des adjectifs « douée » et « habile », mettent en lumière l'importance de la maîtrise de la technique durant les pratiques BDSM. Une fois de plus, à travers la narration de ces scènes, Delorme se fait pédagogue ${ }^{11}$, insistant sur les accessoires et les techniques nécessaires à la réussite des séances BDSM et sur l'importance d'obéir à la directive SSC.

\section{La technique précise du fist-fucking}

Le fist-fucking ${ }^{12}$, considéré parfois ${ }^{13}$ comme une pratique BDSM, nécessite également un savoir-faire précis. Marion raconte une nuit passée avec son ex, Anja, une lesbienne qu'elle a rencontrée sur le site gayvies.com : «Je l'ai baisée par terre sur le matelas [...] et je ne sais pas si elle a joui ou non. Quand ça a été son tour de s'y mettre, elle m'a fait mal en me martelant avec quatre doigts tendus comme si elle s'entraînait à un coup de karaté au lieu de baiser un vagin. Je n'ai pas réussi à jouir » $(\mathrm{G}, 138)$. Contrairement aux scènes BDSM analysées jusqu'à présent et qui soulignent le plaisir et le sentiment de bien-être, on observe ici une tout autre représentation de la pratique sexuelle : elle est douloureuse. Cependant, l'humour du passage ridiculise plutôt la maladresse d'Anja, au lieu de dénoncer le fist-fucking lui-même. Afin de mieux exposer 
l'importance de la maîtrise de la technique, Delorme consacre un chapitre entier au fist-fucking, intitulé «Éloge de la main », dans son recueil Insurrections ! en territoire sexuel. Dans ce chapitre, la narratrice s'adresse à un «tu » anonyme qui pourrait être un·e partenaire ou le lecteur ou la lectrice. Elle utilise des métaphores associées à la navigation pour représenter l'aventure vécue à la fois par elle-même et par la personne dont la main «voyage » dans son vagin : « laisse-moi croire que tu maîtrises la boussole affolée de mes vagues d'endorphines et la cartographie mouvante de ma géographie interne » (EM, 71). D’abord, en utilisant la métaphore de la boussole et de la cartographie mouvante, Delorme fait référence à la sécrétion des hormones de la narratrice lorsqu'elle ressent de l'excitation durant le fist-fucking et au mouvement convulsif de ses muscles vaginaux. Quant à la personne qui fiste, la métaphore de la navigation renvoie au besoin de concevoir une route avant d'arriver à destination, soit à un point précis dans le vagin. Quelques lignes plus loin, la narratrice détaille les possibles défis que peut comporter cette pratique sexuelle, plus précisément au moment où le poing est à l'intérieur du vagin :

Ne va pas plus loin, tu buterais le col de l'utérus et me ferais tordre en déplaisir. Ne sors pas ton poing, tu écartèlerais à nouveau l'entrée du vagin que tu viens de passer. $\mathrm{Tu}$ bouges en piston entre ces deux latitudes sans les dépasser. Si tu forces, je voudrai t'expulser. Viendra un moment où il faudra marteler, mais toujours entre ces deux points d'ancrage.

Si je jouis, je voudrai peut-être te garder repliée en moi un moment comme on berce un petit, peut-être que je pousserai pour t'arroser. Mais ne sors ni trop tôt ni trop tard, tu couperais court au jaillissement. Trop tôt, et je palpite ouverte devant toi, je jouis sans toi à vide, tu m'as ratée. Trop tard, tu me frustres de l'explosion liquide ; ton poignet bouchait l'entrée de l'urètre. (74)

Comme indiqué dans la partie précédente, ce chapitre portant sur le fist-fucking prend également une valeur pédagogique. Delorme utilise explicitement des verbes à l'impératif dans le but de décrire la façon dont la narratrice préfère se faire fister. On remarque aussi que la valeur que prend « marteler » est mise en contraste avec celle du même mot qui se trouve dans l'extrait où il marque plutôt, cette fois-là, la douleur causée par Anja, l'ex de Marion dans Quatrième génération. Ici, l'action de marteler durant le fist-fucking prend une valeur positive tant qu'il est fait avec précision et tant que le vagin n'est pas blessé. De plus, dans «Éloge de la main », en expliquant ce qu'il faut ou ce qu'il ne faut pas faire, Delorme démystifie la pratique du fist-fucking. Autrement dit, celuici n'est ni douloureux ni dangereux si on connaît la technique. Afin de démystifier davantage le fist-fucking, Delorme a recours à un renversement comme stratégie textuelle. 
La narratrice d'Insurrections ! en territoire sexuel décrit une scène de fist-fucking entre elle-même et sa meilleure amie, Marie, et renverse le sujet et l'objet qui « baise »: « au bout de dix minutes que je la fistais, c'est elle qui baisait ma main, qui impulsait le mouvement, qui dirigeait mon bras, mon corps tout entier. Et j'ai pompé pendant une heure, à genoux entre ses jambes écartées »(JVPA, 103 ; je souligne). L'alternance entre les sujets «je » et «elle», de même que celle entre les compléments «la», «mon bras» et «mon corps», pourraient représenter un échange de plaisir, voire une certaine réciprocité. Durant la même scène, le chiasme signale cet échange : «Le fisting avec moi, c'est ta main contre ma chatte. Ou ta chatte contre ma main » (104; je souligne). On constate que le fist-fucking crée une situation intime, telle que décrite par Bauer, dans la mesure où les partenaires se font objets alternativement pour partager une expérience intense commune. Cette intensité est graduellement montrée quand la narratrice se décrit « en symbiose » avec Marie et lorsqu'elle valide son propre sentiment en citant la définition de «symbiose » : «La symbiose [...] c'est une "association durable et réciproquement profitable entre deux ou plusieurs organismes vivants" »(104). Le lendemain, en se rappelant de sa nuit passée avec Marie, la narratrice pense l'aimer « du plus profond [d'elle] » (106) et conclut que l'amour « est partout autour de nous, et d'abord dans nos mains, dans nos bouches, dans nos regards, et dans la chatte de notre meilleure amie » (107). Ainsi, chez Delorme, le fist-fucking est représenté comme une pratique sexuelle qui crée des liens profonds entre les personnes l'exerçant. Dans la prochaine partie, nous verrons qu'il permet aussi d'établir un «safe space » pour les personnes qui souhaiteraient (re)construire leur identité de genre.

\section{Le fist-fucking et son lien avec la construction de l'identité de genre}

Si le fist-fucking a été précédemment représenté entre des personnages féminins, dans Insurrections ! en territoire sexuel Delorme met cette pratique sexuelle en scène entre la narratrice, qui est une femme cis, et des hommes trans. Il arrive qu'au cours des relations BDSM, les adeptes performent des rôles de genre différents de leur identité de genre. Bauer affirme que ces rôles permettent à la personne qui le fait, d'apprendre à être convaincante dans sa performance et de gagner de l'assurance puisque « gender was differently and much less policed within dyke+queer BDSM. Rather, [...] gender [is] a conscious and active co-construction » (197). Chez Delorme, à travers la métaphore de la naissance, cette co-construction de genre est illustrée dans une scène où 
la narratrice aide ses partenaires à construire leur transidentité plutôt que de jouer à un rôle de genre temporaire :

Combien d'entre eux, après que j'ai saisi leurs épaules, caressé leur dos et mordu dans leur nuque, ouvert mes jambes à leurs mains, m’ont dit « Je suis un garçon entre tes bras. » Je les ai mis au monde, les accouchant à l'envers de moi, les laissant venir dans mon ventre d'où ils sortaient plus forts, plus fiers. Ils ont été baptisés Catherine, Nadia ou Nathalie, élevés comme des filles, dans un monde qui décide qui tu es en fonction de ce que tu as entre les jambes à la naissance. (MV, 49 ; je souligne)

Le lexique de la naissance exemplifie non seulement la construction identitaire des trans, mais aussi l'action de la narratrice de leur offrir leur véritable identité. Ensuite, il est intéressant d'observer le renversement de l'accouchement : au lieu de sortir du ventre, les personnages vont à l'intérieur. Cette action met en évidence l'agentivité des personnages trans en ce qui concerne cette co-construction. Plus loin, la narratrice utilise à plusieurs reprises le champ lexical de l'accueil afin de faire ressortir sa propre agentivité quant à la construction identitaire de ses partenaires :

Mon vagin qui accueille sa main, qui s'enveloppe autour de sa chair, lui dit que je nous recrée, moi Ève et lui Adam, même si on est nés tous deux d'anatomie femelle. (50; je souligne)

À toutes ces personnes, qui représentent plus de la moitié de la population terrestre, que dans certains endroits du globe on encense et ailleurs on mutile, écrase et humilie, mon vagin est ouvert. (52; je souligne)

Serrer toutes les mains dans la chair de mon ventre, les accueillir toutes au creux de mon bassin. À l'intérieur de moi, ils sont inconditionnellement bienvenus. (54 ; je souligne)

On se trouve ici devant une autre représentation du fist-fucking qui sert à signifier une position sociopolitique reconnaissant les identités queer et marginalisées. De plus, face aux cruautés de la société, telles que la mutilation, l'écrasement et l'humiliation, la narratrice laisse un message d'espoir à ceux et celles qui veulent se réapproprier leur genre. Enfin, comme le soutient Bauer, le fist-fucking et les pratiques BDSM comprenant des jeux de rôles genrés sont des actes qui non seulement exigent du travail, mais qui s'avèrent aussi « as emotionally rewarding and sexually gratifying, and therefore productive of gender identities and queer desires and pleasures » (198).

\section{Delorme, auteure militante en faveur des sexualités queer}

À côté de certaines féministes qui luttent contre les pratiques BDSM - lesquelles participent au maintien, selon Rubin, de l'oppression des femmes -, d'autres tentent de 
déconstruire les préjugés entourant ces pratiques sexuelles. Quatrième génération et Insurrections! en territoire sexuel offrent justement une représentation positive des pratiques BDSM, une représentation qui s'inscrit dans l'approche féministe pro-sexe et qui vise à infirmer des préjugés. Dans sa représentation de l'ondinisme, par exemple, Delorme n'en fait pas une pratique repoussante, mais plutôt une occasion de vivre une expérience intime avec un·e inconnu·e. De plus, en soulignant les bienfaits des pratiques BDSM à travers un lexique spécifique et mélioratif, l'auteure les valorise. Dans ses ouvrages, Delorme insiste également sur le savoir-faire et la précision nécessaires pour s'adonner à des pratiques BDSM ; qu'il s'agisse du choix des accessoires ou des techniques, la règle est d'obéir à la directive «Safe, Sane and Consensual ». Ainsi, comme pour le fist-fucking, il est essentiel de suivre des étapes et de ne pas blesser les partenaires.

Enfin, on constate que les pratiques BDSM étudiées sont représentées, chez Delorme, de manière à ce que les personnages queer puissent revendiquer et vivre librement leur sexualité. En effet, des féministes, telles qu'Emily Prior, soutiennent que «[a]lthough some [...] women may seem to be engaging in traditional submissive or subordinate sexual roles, BDSM allows women in these roles and in the dominant or top sexual roles to express and experience personal power through their sexual identities » (§ 1). Les pratiques BDSM, selon les féministes pro-sexe, n'ôtent pas le pouvoir aux femmes, elles le leur donnent. Pour conclure, référons-nous à la citation d'Annamarie Jagose en vue de pousser notre réflexion quant à la valeur politique des pratiques sexuelles :

Fist-fucking has taught us a great deal. Among its lessons : the body has the capacity to reterritorialise itself not simply beyond normative, genitally oriented definitions of the sexual, but beyond categories of sexual orientation and sexuality itself [...]. Transformative political potential attaches by default to queer sexual practice, that it is the non-normativity of queer erotic practice that makes it recognisable as political. (521)

Le combat contre la sexualité normative ne cesse pas et Delorme, à travers ses textes, vise à atteindre deux buts : encourager tout sujet à faire de son corps une force politique et donner voix aux personnes dont les pratiques sexuelles sont stigmatisées.

\section{Bibliographie}

Ausina, Anne-Julie. «La performance comme force de combat dans le féminisme ». Recherches féministes 27.2 (2014) : 81-96. 
Bauer, Robin. Queer BDSM Intimacies : Critical Consent and Pushing Boundaries. New York: Palgrave Macmillan, 2014.

Boisclair, Isabelle. «De la théorie dans la création ou la Queer Theory pour les nul-le-s. Les romans de Wendy Delorme ». Loin des yeux près du corps. Entre théorie et création. Dir. Thérèse Saint-Gelais. Montréal : Galerie de l’UQAM/Temue-ménage, 2012 : 123-127.

Califia, Pat. Public Sex : the Culture of Radical Sex. San Francisco : Cleiss, 1994.

Caruso, Jessica. BDSM : les règles du jeu. Montréal : VLB, 2016.

Cruz, Ariane. The Color of Kink : Black Women, BDSM, and Pornography. New York : New York UP, 2016.

Delorme, Wendy. Insurrections ! en territoire sexuel. Vauvert : Au diable Vauvert, 2009.

---. Quatrième génération. Paris : Grasset, 2007.

Dworkin, Andrea. Pornography : Men Possessing Women. New York : Putnam, 1981.

Dworkin, Andrea et Catharine A. MacKinnon. Pornography and Civil Rights : A New Day For Women's Equality. Minneapolis : Organizing Against Pornography, 1988.

Jagose, Annamarie. « Counterfeit Pleasures : Fake Orgasm and Queer Agency ». Textual Practice 24.3 (2010) : 517-539.

Prior, Emily E. «Women's Perspectives of BDSM Power Exchange ». Electronic Journal of Human Sexuality 16 (2013). http://www.ejhs.org/volume16/BDSM.html, consulté le 11 janvier 2018.

Rubin, Gayle. «Thinking Sex ». Deviations : A Gayle Rubin Reader. Durham : Duke UP, [1984] $2011: 137-181$.

Weiss, Margot. Techniques of Pleasure : BDSM and the Circuits of Sexuality. Durham : Duke UP, 2011.

\footnotetext{
Notes

${ }^{1}$ Les chapitres analysés seront désormais désignés par leur sigle respectif. Dans Quatrième génération, «MVT » fait référence à « Mistress Valentine's Toilet », «PFL » à «Post fuck love », «PSM » à «La performance sadomasochiste », «ST» à «Single Tail», « $\mathrm{CG} »$ à «Caligula Party » et « $\mathrm{G} »$ à «gayvies.conne ». Dans Insurrections, «GB » fait référence à «Gang Bang», «ÉM » à «Éloge de la main», «JVPA à «Je suis venue vous parler d'amour » et «MV » à « Métaphysique du vagin ».

${ }^{2}$ Il est vrai que le BDSM est pratiqué par des personnes queer et aussi des personnes qui ne s'identifient pas à la communauté LGBTQ+. Dans le présent article, le terme «BDSM queer » est utilisé pour souligner le fait que dans l'œuvre de Delorme, les adeptes de cette pratique sont queer.

${ }^{3}$ Cette citation de Caruso pourrait semer une certaine confusion quant aux termes «violence », «coercition » et «cruauté », car les pratiques BDSM impliquent le recours à la force physique entre les partenaires. Cependant, ce qui importe est le consentement durant ces pratiques.
} 
${ }^{4}$ Margot Weiss identifie, quant à elle, sept classes de pratiques BDSM : «D/s [...], Bondage [...], Impact [...], Electrical and medical [...], Sex play and sensation [...], Psychological aspects [...], Edge and fetish » (9). Elle affirme que « even if imagined to spring from a core or essential desire, [a BDSM practitioner] requires self-mastery and selfknowledge that is bound to community rules, techniques, and perspectives » (9). Les propos de Caruso reflètent ceux de Weiss et elles soutiennent, toutes les deux, que les pratiques BDSM exigent des méthodes précises.

${ }^{5}$ Il est important de noter que l'ondinisme n'est pas un terme utilisé dans le roman Quatrième génération. En fait, la narratrice n'utilise aucun terme spécifique pour décrire cette pratique sexuelle. Néanmoins, parmi les adeptes de BDSM, cette pratique est souvent connue sous les noms de «piss play », «golden shower » ou « watersports ». Pour mon article, je décide de ne pas employer ces mots, car ils appartiennent au langage familier. L'urolagnie ou l'urophilie sont des synonymes surtout utilisés en médecine. C'est pour cette raison que j'ai choisi « ondinisme », un terme qui renvoie moins à la déviance, et plus à une particularité.

${ }^{6}$ Dans les communautés BDSM, le/la top est la personne qui domine, alors que le/la bottom est celle qui est soumise. Dans d'autres cas, le/la top est aussi appelé(e) le/la sadique, tandis que le/la bottom est le/la masochiste (Califia 158).

${ }^{7}$ Bauer soutient qu'avant une séance BDSM, certains adeptes ressentent une anxiété (performance anxiety) (63), car certains détails sont essentiels à son bon déroulement, tels que boire trois litres d'eau et uriner au bon moment dans le cas de Marion. L'anxiété ne vient vraisemblablement pas d'une inquiétude de se faire juger par les partenaires, mais de celle de ne pas réussir à leur donner du plaisir. Au sein des communautés BDSM, les adeptes ne se contentent pas de recevoir du plaisir, ils et elles cherchent aussi à en procurer.

${ }^{8}$ Chez Delorme, certains personnages féminins portent des noms qui sont le plus souvent associés au masculin et c'est l'une des stratégies que l'auteure emploie pour renverser les stéréotypes liés aux genres sexuels.

${ }^{9}$ La notion de douleur, explorée dans le livre de Bauer, pourrait être pertinente pour l'analyse des pratiques BDSM chez Delorme. Cependant, puisque la douleur passe au second plan, après la valeur spirituelle des pratiques, elle n'est pas examinée dans mon article. Ce qu'il faut retenir du texte de Bauer, c'est le fait que la douleur prend une autre signification lorsqu'elle est attendue et subie dans un contexte spécifique (166).

${ }^{10}$ Dans son étude approfondie des pratiques BDSM, Bauer offre un point de vue contraire à d'autres théoriciens, tels que Weiss (2011), qui soutiennent que les scènes d'esclaves/maîtres trivialisent le passé des esclaves et le racisme. Bauer affirme que : «this danger seems to be inherent in the extensive use of the term "slave," which is circulated widely in the BDSM communities as a BDSM-specific technical term, so that its meaning is shifted in a way that seems to disassociate it from its racist history politically, while at the same time exploiting the emotional attachments to this history for sexual pleasures » (188). Selon lui, certains termes employés parmi les adeptes du BDSM ont un sens autre que celui défini par la majorité de la société. Nous reconnaissons toutefois que les scènes d'esclaves/maîtres restent problématiques, notamment parmi les couples interraciaux (Weiss 196). À propos des adeptes du BDSM dans les communautés noires, Ariane Cruz écrit : « [...] whether black women can indeed consent to racialized sexual play, black women BDSMers suggest that this consent is not only possible but also pleasurable and affectively empowering » (45). La question du consentement est alors primordiale dans les pratiques BDSM.

${ }^{11}$ Isabelle Boisclair remarque judicieusement que Delorme «se plait à diffuser des savoirs [...] et à les vulgariser, les rendant accessibles à un plus grand nombre. D'une certaine façon, on peut dire de ses textes qu'ils constituent de parfaits manuels de Queer Theory $101 »(123)$.

${ }^{12}$ Comme le terme l'indique en anglais, cette pratique consiste à insérer le poing dans le vagin ou dans l'anus. Le « fist-fucking » est, contrairement au terme « ondinisme », lui-même employé par Delorme.

${ }^{13}$ Puisque cette pratique peut se faire seule ou à plusieurs, ou hors d'une séance BDSM, nous ne pouvons la catégoriser exclusivement ainsi. 